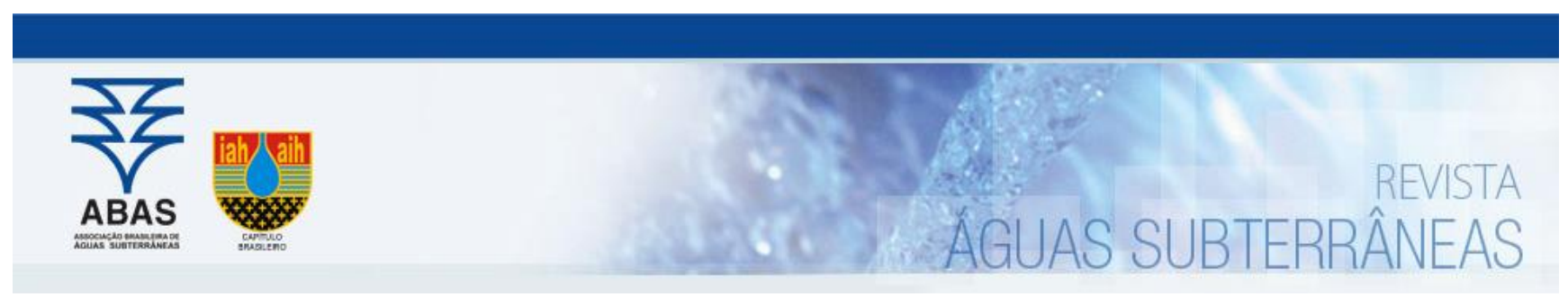

Artigos

\title{
Sorção da sulfadiazina em solo urbano: experimentação e modelagem
}

\section{Sorption of sulfadiazine in urban soil: experimentation and modeling}

\author{
Emidio Dias Silva Júnior1; Artur Paiva Coutinhow2; Severino Martins dos Santos Neto1; Ana Emilia Carvalho de Gusmão da

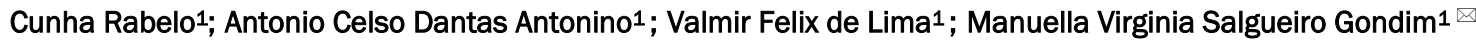 \\ 1 Universidade Federal de Pernambuco (UFPE), Centro de Tecnologia e Geociências - Escola de Engenharia de Pernambuco \\ (CTG-EEP), Departamento de Energia Nuclear, Recife, PE \\ 2 Universidade Federal de Pernambuco (UFPE), Campus do Agreste, Núcleo de Tecnologia, Caruaru, PE
}

\section{$\triangle$ emidiojuniorduo@hotmail.com, arthur.coutinho@yahoo.com.br, martinsdsn@gmail.com, anaerabelo@gmail.com, acda@gmail.com, atrazina@gmail.com, manuellavirginia@yahoo.com.br}

Palavras-chave:
Isoterma de sorção.
Cinética de sorção.
Antimicrobiano.
Contaminante de preocupação emergente.

Keywords:

Sorption isotherm.

Sorption kinetics.

Antibiotic.

Emergent pollutant.

Revisado por pares.

Recebido em: 02/05/2019.

Aprovado em: 02/09/2019.

\section{Resumo}

A interação dos poluentes na zona vadosa é fundamental para determinação do grau de vulnerabilidade da água subterrânea quando do transporte de contaminantes no solo. 0 presente trabalho apresenta a interação do antimicrobiano sulfaziadina (SDZ) em solo urbano na cidade do Recife. A cinética e isoterma de sorção da SDZ foram determinadas em quatro profundidade de um perfil de solo urbano. As concentrações da sulfadiazina foram determinadas por cromatografia líquida de alta eficiência (HPLC) com detecção por absorção ultravioleta. Os estudos de cinética demonstraram que a sorção foi rápida nas primeiras dez horas do período de contato entre o solo e a solução da SDZ. 0 equilíbrio foi alcançado com 24 horas de interação nas quatro camadas. 0 modelo de segunda ordem apresentou os melhores ajustes da cinética de sorção em todas as profundidades com valores de $\mathrm{R} \neg 2$ entre 0,94 a 0,98. 0 modelo que melhor se adequou na representação das isotermas de sorção foi o modelo Linear. Os baixos valores de KD (1,3 a 1,9 L.kg-1) indicam alta mobilidade e elevado risco de contaminação das águas subterrâneas por esse composto.

\section{Abstract}

The evaluation of the interaction of pollutants in the vadose zone is fundamental for determining the degree of vulnerability of groundwater when transporting contaminants in the soil. The present work presents the interaction of an antibiotic of the type Sulfaziadina in urban soil in the city of Recife. The kinetics and sorption isotherm of Sulfadiazine (SDZ) were determined in a sandy-loam soil of the urban area of the city of Recife. Sorption kinetics and sorption isotherms were performed in for layers of an urban soil profile. Sulfadiazine concentration was determined by high performance liquid chromatography (HPLC). The kinetics studies showed that the sorption was rapid in the first ten hours of the contact period between the soil and the SDZ solution. The equilibrium was reached with 24 hours if interaction in 04 layers. For this soil, SDZ sorption kinetics were reasonably described by a first order model.The second order kinect model presented the best adjustments of sorption kinetics at all depths with R2 values between 0.94 and 0.98 . The model that best fit the representation of sorption isotherms was the Linear model. The low KD values (1.3 to 1.9 L.kg1) indicate high mobility and high risk of groundwater contamination by this compound.

DOI: http:/dx.doi.org/10.14295/ras.v33i4.29543

\section{INTRODUÇÃO}

A interação dos poluentes na zona vadosa é fundamental para estudos da vulnerabilidade da água subterrânea, quando do transporte de contaminantes no solo. 0 transporte de poluentes para as águas superficiais é diretamente influenciado pelo processo de sorção. Por sua vez, o comportamento da sorção varia de acordo com as propriedades físico-químicas como o coeficiente de partição $\mathrm{K}_{\mathrm{D}}$, as propriedades da matriz sólida do solo, como o teor de matéria orgânica e tipo de minerais do solo, além de fatores ambientais como $\mathrm{pH}$ e temperatura (PAVLOVIc et al., 2018; BOY-ROURA et al., 2018). O Kd é uma medida direta da partição da molécula do poluente entre as fases sólidas e líquida, constituindo um parâmetro de referência, se tornando um pré-requisito importante para a avaliação de risco do lençol freático (SRINIVASAN, SARMAH, 2014; Ternes et al., 2015).

Antibióticos são largamente utilizados para prevenir infecções bacterianas, porém esses compostos não são totalmente metabolizados pelo organismo humano e animal, onde 30\% a 90\% são excretados via urina e fezes na forma inalterada (CARVALHO \& SANTOS, 2016; XU et al., 2015). As sulfonamidas (SAs) são uma das classes de drogas antibacterianas mais utilizadas na medicina humana e veterinária (SUKUL \& SPITELLER, 2006; HU et al., 2010; JOHNSON et al., 2015; 
ZHANG et al., 2017). Desta classe, a sulfadiazina (SDZ) é uma das que apresenta mais altas concentrações no ambiente, dada a sua acentuada aplicação (YAN et al., 2013), resultando em concentrações de 17 ng.L-1 em águas subterrâneas (KÜMMERER, 2009) e 505 ng.L-1 em águas superficiais (Bu et al., 2013), gerando riscos ambientais (CONDE-CID et al., 2019).

Deste modo, estes compostos podem entrar no ambiente natural através da descarga direta de efluentes das Estações de Tratamento de Esgoto, onde são ineficientemente removidos (GOBEL et al., 2007; RODRIGUEZ-MOZAZ et al., 2015; HE et al., 2015). Dan et al. (2013) ressaltam que a eficiência de remoção da Sulfadiazina em estações de tratamentos é de aproximadamente $50 \%$. Nos centros urbanos do Nordeste o acesso ao saneamento básico é deficitário onde somente $55,1 \%$ dos municípios apresenta rede de coleta de esgoto (IBGE - Atlas de Saneamento, 2011). Em Recife o atendimento chega a ser de apenas 35\% (IBGE-Estatísticas, 2010).

Nesse contexto, o lançamento de esgoto sem tratamento é uma das principais portas de entrada dos contaminantes emergentes no meio ambiente. Corroborando para a elevação do risco de contaminação, uma vez que os antibióticos podem ser sorvidos ao solo, transportados para as águas superficiais e lixiviados para os lençóis freáticos (DORETTO \& RATH, 2013; SRINIVASAN e SARMAH, 2014; YANG et al., 2018).

Diante do exposto, é de fundamental importância o entendimento dos processos e mecanismos que vão resultar na mobilidade e retenção da SDZ na zona não saturada. Desta forma, o objetivo desse trabalho é avaliar o processo de sorção do antibiótico sulfadiazina (SDZ), em um solo de aterro urbano da cidade do Recife, com o intuito de avaliar a vulnerabilidade do aquífero.

\section{MATERIAL E MÉTODOS}

\section{1. Área de Estudo}

A área de estudo está localizada na cidade do Recife, estado de Pernambuco. Mais precisamente na margem do Riacho do Cavouco que corta o Centro de Tecnologias e Geociências no campus da Universidade Federal de Pernambuco (UFPE), latitude $8^{\circ} 03^{\prime} 1$ " e longitude $34^{\circ} 52^{\prime} 52^{\prime \prime}$ (Figura 1).

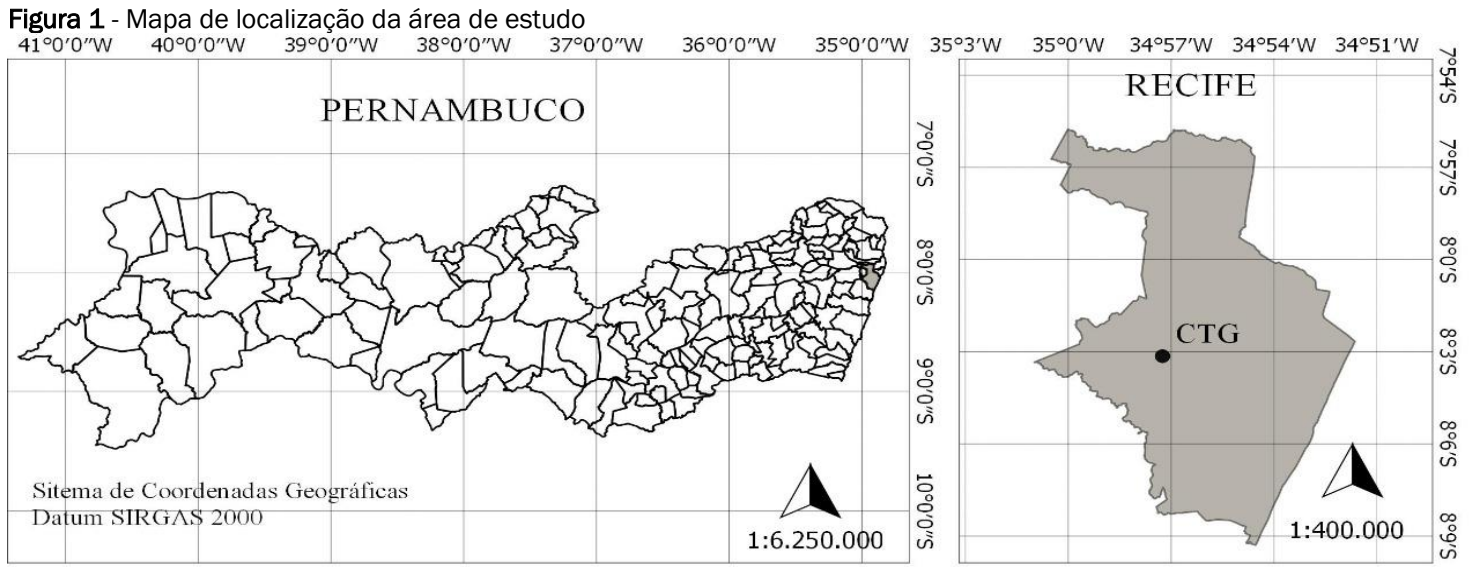

\subsection{Caracterização físico-química do solo}

$\mathrm{Na}$ área de estudo foram coletadas amostras deformadas de solo em quatro profundidades diferentes $(0-20 \mathrm{~cm} ; 20-40 \mathrm{~cm}$; $40-60 \mathrm{~cm}$ e $60-80 \mathrm{~cm}$ ) de um mesmo perfil de solo. Para cada profundidade foram retiradas aproximadamente $5 \mathrm{~kg}$ de solo. As amostras de solo passaram por secagem ao ar por aproximadamente 72 horas, foram destorroadas e passadas em peneira de $2 \mathrm{~mm}$.

A análise granulométrica foi realizada em triplicata para cada profundidade, sendo que a fração grossa foi determinada por peneiramento e os teores de argila e silte foram determinados por sedimentação (EMBRAPA, 1997). 0 pH foi determinado em água e em $\mathrm{KCl}$ a $1 \mathrm{~mol} . \mathrm{L}^{-1} \mathrm{com}$ um potenciômetro e suspensão solo:líquido (água, $\mathrm{KCl}$ ) na proporção de 1:2,5 m/v.

O carbono orgânico (COT) e a matéria orgânica (MO) foram determinados pela metodologia Walkley-Black modificado, sendo a porcentagem de MO calculada multiplicando-se o COT pelo fator 1,724 , o qual admite que o carbono participa com 58\% na composição média dos húmus (EMBRAPA, 1997).

\subsection{Cinética de sorção da sulfadiazina (SDZ)}

A sulfadiazina (SDZ) é um antibiótico da familia das sulfonamidas e possui massa molar 250,28 g.mol-1, Log Kow - 0,09 e é uma molécula ionizável com dois prótons trocáveis $\left(\mathrm{pKa}_{1}=1,57 ; \mathrm{pKa}_{2}=6,5\right)$ (SUKUL et al., 2008).

A cinética de sorção foi avaliada partindo de uma solução contendo $\mathrm{CaCl}_{2}$ a 0,01 mol e SDZ com concentração de $30 \mathrm{mg} . \mathrm{L}^{-}$ 1. A relação solo/solução foi de $1: 10 \mathrm{~m} / \mathrm{v}$ ( $5 \mathrm{~g}$ de solo para 50 $\mathrm{mL}$ de solução de SDZ). Os recipientes foram colocados em mesa agitadora e em intervalos de tempo preestabelecidos $(0$; 0,$5 ; 1 ; 2 ; 3 ; 4 ; 6 ; 8 ; 10 ; 12 ; 24 ; 36 ; 48$ h) e coletadas alíquotas de $1 \mathrm{~mL}$. Após a centrifugação o sobrenadante foi filtrado, em triplicata, em filtro de acetato de celulose com 0,45 $\mu \mathrm{m}$ de porosidade. A concentração da SDZ no sobrenadante foi determinada por cromatografia líquida de alta eficiência 
(HPLC-UV). As condições cromatográficas foram coluna Phenomenex C18 (250 mm x $46 \mathrm{~mm} ; 5 \mu \mathrm{m}$ ), e fase móvel composta de água:ácido fórmico:metanol (64,5:0,5:35 v/v/v), com vazão de $1,0 \mathrm{~mL}$. min-1. A detecção foi por absorção no ultravioleta, com comprimento de onda de $265 \mathrm{~nm}$.

\subsection{Isoterma de sorção}

A relação solo-solução foi de 1:10 m/v, contendo SDZ nas concentrações $\left(\mathrm{C}_{0}\right): 10,15,20,25,30,35 \mathrm{mg} \mathrm{L}$-1. As amostras foram preparadas em triplicata, agitadas por $48 \mathrm{~h}$ em mesa agitadora a $200 \mathrm{rpm}$ e centrifugadas a $10.000 \mathrm{rpm}$ por 10 min. O sobrenadante foi filtrado e analisado por CLAE, obtendo-se pela Equação 1 a quantidade de SDZ sorvida ao solo.

$$
S=\left(C_{0}-C_{e}\right) \cdot F D
$$

Onde:

S - quantidade de SDZ sorvida pelo solo (mg.kg-1);

$\mathrm{C}_{0}$ - concentração de SDZ da solução colocada em contato com o solo (mg.t-1);

Ce - concentração de SDZ na solução após o equilibrio (mg.L-1);

FD - fator de diluição considerando a relação solução/solo (no caso, $\mathrm{FD}=50 / 5=10$ ).

\subsection{Modelagem da sorção}

A cinética de sorção foi determinada pelos modelos de primeira e segunda ordem, e difusão intra-partícula (YANEVA; KOUMANOVA, 2006). A isoterma de sorção foi calculada pelos modelos lineares e não lineares de Freundlich e Langmuir (Liu et al., 2017). As equações utilizadas são apresentadas na Tabela 1.

Tabela 1 - Modelos utilizados para representar a isoterma e a cinética de sorção

\begin{tabular}{ccc}
\hline & Cinética de Sorção & \\
\hline Modelo de 1' Ordem & Modelo de $2^{\circ}$ Ordem & $\begin{array}{c}\text { Modelo de Difusão } \\
\text { Intra-partícula } \\
S_{t}=k_{i} t^{0,5}\end{array}$ \\
\hline $\log \left(S_{e l}-S_{t}\right)=\log S_{e l} p-\frac{k 1}{2,303} t$ & $\frac{t}{S_{t}}=\frac{1}{k_{s}}+\frac{1}{S_{e 2}} t \operatorname{com} k_{S}=k_{2} S_{e 2}^{2}$ & \\
\hline Modelo Linear & Isoterma de Sorção & Modelo Langmuir \\
$S=K_{D} C$ & Modelo Freundlich & $S=\frac{K_{L} S_{0} C_{e}}{1+K_{L} C_{e}}$ \\
\hline
\end{tabular}

Onde:

S - fração da substância sorvida (mg.kg-1);

C - concentração da substância na fase líquida (mg.L-1);

$\mathrm{K}_{\mathrm{D}}$ - coeficiente de partição solo-solução (L.kg-1);

$\mathrm{K}_{\mathrm{F}}$ - coeficiente de Freundlich $\left(\mathrm{kg}^{-1} \mathrm{mg}^{1-1 / n} \mathrm{~L}^{1 / n}\right)$;

$\mathrm{K}_{\mathrm{L}}$ - coeficiente de Langmuir (L.mg-1);

So - capacidade máxima de sorção (mg.kg-1);

$\mathrm{S}_{\mathrm{el}}$ - capacidade de sorção em equilíbrio $\left(\mathrm{mg}^{\mathrm{kg}} \mathrm{kg}^{-1}\right)$;

$\mathrm{S}_{\mathrm{t}}$ - capacidade de sorção no tempo (mg.kg-1);

$\mathrm{k}_{1}$ - taxa constante de sorção de primeira ordem $\left(\mathrm{h}^{-1}\right)$;

$\mathrm{S}_{\mathrm{e} 2}$ - capacidade de sorção em equilíbrio para o modelo de segunda ordem (mg.kg-1);

$\mathrm{k}_{2}$ - taxa constante de sorção de segunda ordem (kg.mg-1. $\mathrm{h}^{-1}$ ); ks - taxa inicial de sorção (mg.kg-1. $\mathrm{h}^{-1}$ );

$\mathrm{k}_{\mathrm{i}}$ - taxa constante de difusão intra-partícula (mg.kg-1.'. $\left.\mathrm{h}^{-1}\right)$.

Três critérios estatísticos foram utilizados para análise dos dados calculados pelos modelos de primeira e segunda ordem: A razão de desvios, $R D$ (Equação 2); coeficiente de determinação, $R^{2}$ (Equação 3 ); coeficiente de massa residual, CMR (Equação 4).
$R D=\frac{\sum_{i=1}^{N}\left(M_{i}-\bar{M}\right)^{2}}{\sum_{i=1}^{N}\left(T_{i}-\bar{M}\right)^{2}}$

$R^{2}=1-\frac{\sum_{i=1}^{N}\left(T_{i}-M_{i}\right)^{2}}{\sum_{i=1}^{N}\left(M_{i}-\bar{M}\right)^{2}}$

$C M R=\frac{\sum_{i=1}^{N} M_{i}-\sum_{i=1}^{N} T_{i}}{\sum_{i=1}^{N} M_{i}}$

Onde:

$T_{i}$ - valores calculados pelo modelo;

$M_{i}$ - valores experimentais;

$\bar{M}$ - média dos valores experimentais;

$\mathrm{N}$ - número de determinações.

\section{RESULTADOS E DISCUSSÃO}

\subsection{Características físico-químicas do solo}

Os solos estudados apresentam textura do tipo areia franca e franco arenosa com percentual de areia, variando de 72,6 a $81 \%$. 0 percentual de argila não ultrapassa $11,4 \%$ conforme demonstrado na Tabela 2. 
Tabela 2 - Características físico-químicas do solo urbano na região metropolitana do Recife

\begin{tabular}{|c|c|c|c|c|c|}
\hline & & \multicolumn{4}{|c|}{ Profundidade $(\mathrm{cm})$} \\
\hline & & $0-20$ & $20-40$ & $40-60$ & $60-80$ \\
\hline \multicolumn{2}{|c|}{ Areia } & $81,80 \%$ & $72,60 \%$ & $74,10 \%$ & $76,20 \%$ \\
\hline \multicolumn{2}{|c|}{ Silte } & $7,5 \%$ & $17,4 \%$ & $15,9 \%$ & $12,4 \%$ \\
\hline \multicolumn{2}{|c|}{ Argila } & $10,7 \%$ & $10,0 \%$ & $10,0 \%$ & $11,4 \%$ \\
\hline \multicolumn{2}{|c|}{ Textura } & Areia Franca & Franco Arenoso & $\begin{array}{c}\text { Franco Are- } \\
\text { noso }\end{array}$ & Franco Arenoso \\
\hline \multirow{2}{*}{$\mathrm{pH}$} & $\mathrm{H}_{2} \mathrm{O}$ & 6,3 & 6,4 & 6,8 & 6,9 \\
\hline & $\mathrm{KCl}$ & 5,4 & 5,9 & 5,9 & 5,9 \\
\hline \multicolumn{2}{|c|}{ COT (g.kg-1) } & 9,8032 & 10,912 & 9,328 & 5,1392 \\
\hline \multicolumn{2}{|c|}{$\mathrm{MO}\left(\mathrm{g}^{\mathrm{kg}} \mathrm{g}^{-1}\right)$} & 16,900 & 18,812 & 16,081 & 8,859 \\
\hline
\end{tabular}

*COT (Carbono Orgânico Total); MO (Matéria Orgânica)

O teor de carbono orgânico (COT) e a matéria orgânica (MO) estão apresentados na Tabela 2. A camada 20-40 foi a que apresentou os maiores valores de COT $(10,912$ g.kg-1) e MO $(18,812$ g.kg-1), isto se deve provavelmente as heterogeneidades comumente encontradas em solos de aterros.

Uma avaliação confiável da mobilidade das sulfonamidas passa pela identificação dos fatores que afetam a sorção desse poluente para os solos e sedimentos (Doretto \& Rath, 2013). A informação dos teores de argila, carbono orgânico e matéria orgânica são fundamentais pois privilegiam o processo de retenção. Thiele-Bruhn et al. (2004) e Doretto \& Rath (2013) sugerem que para a sorção de sulfonamidas no solo o conteúdo de matéria orgânica, a presença de minerais argilosos e de óxidos são importantes.

Ainda de acordo com a Tabela 2, os valores do $\mathrm{pH}$ do solo pouco variaram entre as amostras das profundidades analisadas. $\mathrm{O} \mathrm{pH}$ em água variou de 6,3 a 6,9 , sendo classificado como levemente ácido.

Solos que apresentam, em água, tendências de valores de $\mathrm{pH}$ maiores que 6 , influenciam na desprotonação da molécula e repulsão eletrostática na presença de coloides do solo carregados negativamente (THIELE-BRUHN, 2003; SUKUL \& SPITELLER, 2006).

As sulfonamidas são pouco solúveis em água, comumente esses antimicrobianos se comportam como ácidos orgânicos fracos e formam sais em solução fortemente ácida ou básica, quando em pH ácidos (DORETTO \& RATH, 2013).

$\mathrm{O} \mathrm{pH}$ ambiental na faixa de 3,5 a 8,0 demostra que há um favorecimento na formação de moléculas na forma neutra, gerando uma hipótese de que outros mecanismos além das interações eletrostática podem influenciar a sorção do SDZ (REIA, 2013). A SDZ é afetada pelo pH, com valores entre 1,57 a 6,50, ionizando espécies catiônicas e aniônicas de acordo com o respectivo aumento do $\mathrm{pH}$, o que pode ocasionar uma diminuição da sorção da SDZ (DORETTO \& RATH, 2013).

\subsection{Cinética de Sorção}

A cinética de sorção para as quatro profundidade analisadas e os respectivos ajustes com a cinética de primeira ordem e segunda ordem são demonstradas na Figura 2.

Observa-se que o equílibrio entre o SDZ em solução e o sorvido nos solos foi alcançado após 24 horas de contato entre solo e a solução para as quatro camadas. Para a camada $0-20 \mathrm{~cm}$

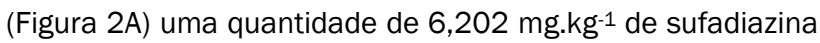
foi sorvida no início do experimento e na ordem de 54 mg.kg-1 na saturação.

Para a camada de 20 - $40 \mathrm{~cm}$ (Figura 2B) a quantidade em equilíbrio foi na ordem de $38 \mathrm{mg} . \mathrm{kg}^{-1}$. A camada de $40-60$ $\mathrm{cm}$ (Figura $2 \mathrm{C}$ ) foi capaz de reter $24 \mathrm{mg} . \mathrm{kg}^{-1}$ de sufadiazina. A camada mais profunda de $60-80 \mathrm{~cm}$ (Figura 2D) em equilíbrio foi observada uma sorção em torno de 25 mg.kg-1.

Analizando o estudo desenvolvido por Sukul \& Spiteller (2006) verifica-se que o valor alcançado no equilíbrio de sorção estava diretamente relacionado às umidades volumetricas na saturação. Assim, o solo que apresentou maior umidade volumétrica na saturação ou maior porosidade foi também o que apresentou maior sorção no equilíbrio.

Além disso, observa-se que a sorção se divide em dois estágios, aumentando rapidamente na primeira interação da SDZ com o solo e após algumas horas esse processo vai ocorrendo mais lentamente até alcançar o equilíbrio, que foi observado às $24 \mathrm{~h}$ (Figura 2 (A), (B), (C) e (D)). Comportamento semelhante foi obtido por Peruchi et al. (2015) em seu estudo com norfloxacina e por Pavlovic et al. (2018) em análise com o albendazol. 0 tempo de equilíbrio de 24 horas também foi demonstrado em estudos semelhantes conduzidos com a SDZ, além de outras sulfonamidas, tais como a sulfadimetoxina e o sulfametoxazol (LEAL et al., 2013; PARK \& HUWE, 2016; SHEN et al., 2018). 
Figura 2 - Modelos de primeira e segunda ordem da cinética de sorção da profundidade de (A) 0 - $20 \mathrm{~cm}$, (B) 20 - $40 \mathrm{~cm}$, (C) $40-60 \mathrm{~cm}$

A) e (D) 60 - $80 \mathrm{~cm}$

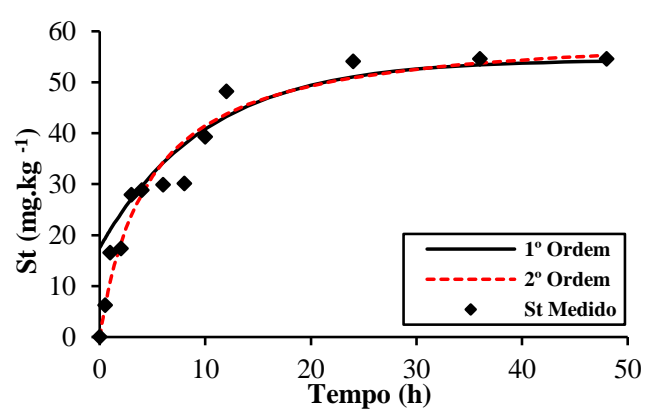

C)

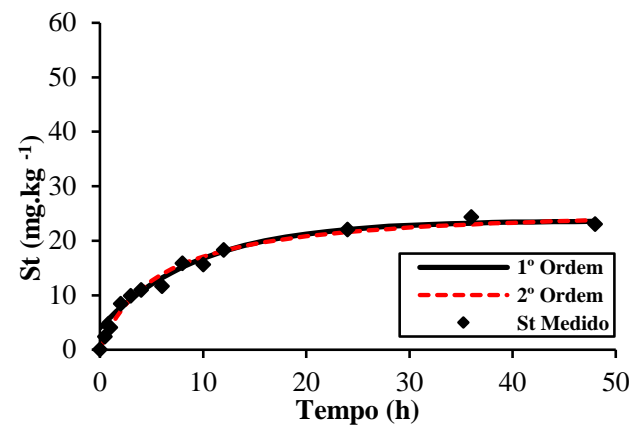

No estudo de Leal et al. (2013), a sorção de cinco sulfonamidas diferentes, entre elas da SDZ alcançou o equilíbrio aparente com 24h. Park \& Huwe (2016) estudaram a sorção de SMX, sulfametoxina e sulfametazina em dois solos arenosos, variando as concentrações entre $0,01 \mathrm{mg}^{-L^{-1}}$ até 5 mg.L-1 e utilizaram o tempo de 24 horas para alcançar o equilíbrio.

Para os ensaios da isoterma de sorção Shen et al. (2018) estudaram a sorção de diferentes antimicrobianos do tipo
B)

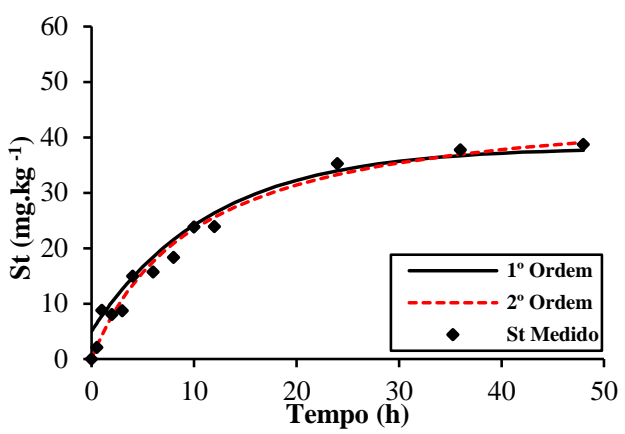

D)

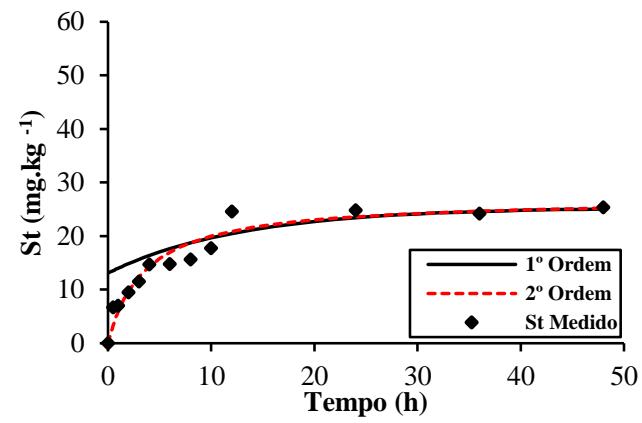

sulfanamidas, em um solo de superfície proveniente de áreas agrícolas. Os ensaios de isotermas foram efetuados considerando concentrações diferentes, que variaram de 0,5 a $10 \mathrm{mg} \cdot \mathrm{L}^{-1} \mathrm{e}$ o tempo de equilibrio de 24 horas.

No tocante aos modelos de sorção de primeira e segunda ordem, na Tabela 3 são apresentados os parâmetros estatísticos que resumem a qualidade dos ajustes.

Tabela 3 - Coeficiente de determinação, coeficiente de massa residual e razão dos desvios para os modelos de $1^{\circ}$ e $2^{\circ}$ ordem.

\begin{tabular}{|c|c|c|c|c|c|c|}
\hline \multirow[b]{2}{*}{ Prof $(\mathrm{cm})$} & \multicolumn{3}{|c|}{ Primeira Ordem } & \multicolumn{3}{|c|}{ Segunda Ordem } \\
\hline & $\mathrm{R}^{2 *}$ & $\mathrm{RD}^{\mathrm{a}}$ & $\mathrm{CMR}^{\mathrm{b}}$ & $\mathrm{R}^{2}$ & RD & CMR \\
\hline $0-20$ & 0,9416 & 2,913 & $-0,1133$ & 0,957 & 0,986 & 0,0054 \\
\hline $20-40$ & 0,9815 & 1,189 & $-0,084$ & 0,9784 & 0,989 & 0,0027 \\
\hline $40-60$ & 0,9743 & 1,235 & $-0,0478$ & 0,9843 & 0,991 & $-0,0026$ \\
\hline $60-80$ & 0,892 & 2,175 & $-0,2065$ & 0,9415 & 0,952 & 0,0039 \\
\hline
\end{tabular}

Observa-se que para o modelo de primeira ordem os coeficientes de determinação $\left(R^{2}\right)$ foram superiores a 0,8920, embora os valores da razão de desvio (RD) se desviaram significativamente do valor de referência. A Razão de desvio (RD) descreve a razão entre o espalhamento dos valores calculados pelo modelo teórico correspondente, o valor esperado tende a 1.

Com relação aos valores do coeficiente de massa residual (CMR), verificou-se que o modelo de segunda ordem apresenta melhor desempenho que o modelo de primeira ordem. Isso demonstra ausência de desvios sistemáticos entre valores teóricos e experimentais.
Uma análise global dos parâmetros estatísticos indica que o modelo cinético de primeira ordem foi razoável, contudo, o modelo de segunda ordem é o mais adequado para representar a cinética de sorção dos solos estudados e a SDZ. Segundo Ho e Mckay et al. (1999) e Liu et al. (2017) isso indica que foi um processo de quimissorção, envolvendo compartilhamento ou troca de elétrons entre o sorvente e o sorbato.

A aplicação do modelo de difusão intra-partícula aos dados das cinéticas de sorção da SDZ para as quatro profundidades do solo são apresentadas na Figura 3. A relação funcional entra a capacidade de sorção $\left(S_{t}\right)$ e a raiz quadrada do tempo $\left(t^{0,5}\right)$, demonstrou dois comportamentos distintos para todas as camadas. 
Figura 3 - Aplicação do modelo de difusão intrapartícula aos dados das cinéticas de sorção para o perfil de solo urbano na cidade do Recife

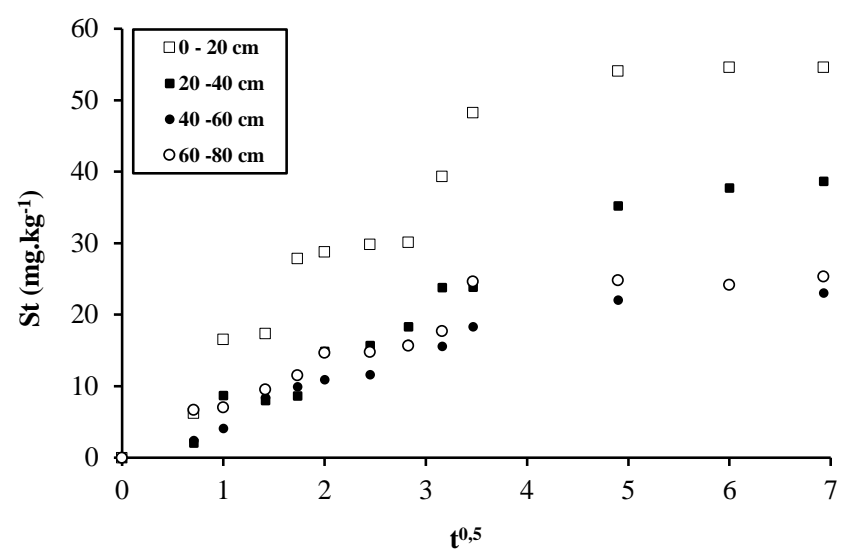

Os poros compõem a estrutura natural do solo e podem ser subdivididos em macroporos e microporos. As curvas da Figura 3, demonstram a difusão que ocorre nos macroporos (segmento com maior declividade) e nos poros de transição (segmento de menor declividade). Atribuindo um $\mathrm{k}_{i}$ específico a cada segmento, pode-se considerar que o $k_{i 1}$ para os macrosporos são maiores que o $k_{i 2}$ para os poros de transição. Isto ocorre pela obstrução ou pela diminuição dos camihos livres disponíveis para difusão ou pelo entupimento dos poros (MILFONT et al., 2006).

Analisando o gráfico da Figura (3), é possível observar inicialmente um enorme contraste entre a curva da camada

superfícial e as demais curvas. A variabilidade entre as profundidade de $20-40 \mathrm{~cm}, 40-60 \mathrm{~cm}$ e $60-80 \mathrm{~cm}$ é pequena. 0 comportamento apresentado pelas camadas de $40-60 \mathrm{~cm}$ e $60-80 \mathrm{~cm}$ é bem parecido. Isso pode ser notado pela proximidade entre os coeficientes de difusão intrapartícula dessas camadas.

Os resultados da aplicação do modelo cinético de primeira ordem, do modelo cinético de segunda ordem e do modelo de difusao-intrapartícula (taxas constantes de sorção, k1, k2 e ki; e das capacidades de sorção em equilíbrio, $S_{e l}$ e $S_{e 2}$ ) são apresentados na Tabela 4.

Tabela 4 - Valores das capacidades de sorção em equilíbrio, $\mathrm{Se}_{1}$ e $\mathrm{Se}_{2}$; das taxas constantes de sorção, $\mathrm{k}_{1}$, $\mathrm{k}_{2}$ e $\mathrm{k}_{\mathrm{i}}$; e dos coeficientes de determinação, $R^{2}$ para as camadas $(0-20 \mathrm{~cm}, 2-40 \mathrm{~cm}, 40-60 \mathrm{~cm}$ e $60-80 \mathrm{~cm})$ do solo urbano na cidade do Recife

\begin{tabular}{|c|c|c|c|c|c|}
\hline \multirow{3}{*}{$\begin{array}{l}\text { Prof. } \\
(\mathrm{cm})\end{array}$} & \multicolumn{2}{|c|}{$1^{\circ}$ Ordem } & \multicolumn{2}{|c|}{$2^{\circ}$ Ordem } & \multirow{3}{*}{$\begin{array}{c}\text { Difusão intra-partícula } \\
\begin{array}{c}\mathbf{k}_{\mathbf{i}} \\
\text { (mg.kg-1.h-1) }\end{array} \\
S_{t}=k_{i} t^{0,5}\end{array}$} \\
\hline & $\begin{array}{c}k_{1} \\
\left(h^{-1}\right)\end{array}$ & $\begin{array}{c}\mathrm{Se}_{1} \\
\left(\mathrm{mg} \cdot \mathrm{kg}^{-1}\right)\end{array}$ & $\begin{array}{c}\mathrm{k}_{2} \\
\left(\mathrm{~kg} \cdot \mathrm{mg}^{-1} \mathrm{~h}^{-1}\right)\end{array}$ & $\begin{array}{c}\mathrm{Se}_{2} \\
\left(\mathrm{mg} \cdot \mathrm{kg}^{-1}\right)\end{array}$ & \\
\hline & \multicolumn{2}{|c|}{$\frac{d S_{t}}{d t}=k_{1}\left(S_{e 1}-S_{t}\right)$} & \multicolumn{2}{|c|}{$k_{S}=k_{2} S_{e 2}^{2}$} & \\
\hline $0-20$ & 0,100 & 36,88 & 0,003 & 60,61 & 10,170 \\
\hline $20-40$ & 0,085 & 33,16 & 0,002 & 47,39 & 6,354 \\
\hline $40-60$ & 0,104 & 19,70 & 0,006 & 26,52 & 4,253 \\
\hline $60-80$ & 0,077 & 12,26 & 0,010 & 27,10 & 4,779 \\
\hline
\end{tabular}

Analisando a Tabela 4, a taxa de difusão intrapartícula é decrescente com a profundidade. Observa-se que para as camadas franco-arenosas, isto é, para as camadas a partir de 20-40 cm, os coeficientes de difusão intrapartícula apresentaram relação direta com os percentuais de (argila + silte) e com o percentual de MO.

\subsection{Isotermas de Sorção}

A análise das isotermas de sorção da sulfadiazina, nos solos das quatro profundidades,é uma importante contribuição para a estimativa do risco de contaminação das águas subterrâneas. Para os solos estudados, as isotermas representam a quantidade de sulfadiazina sorvida por unidade de massa de solo em função da concentração de equilíbrio. Os dados experimentais foram ajustados para o modelo Linear, o modelo de Freundilich e o modelo de Langmuir (Tabela 1). Na Figura 4 são apresentadas as isotermas de sorção dos solos estudados da zona urbana do Recife.

O valor do coeficiente de partição solo-solução linear $\left(K_{D}\right)$ foi obtido por uma regressão linear. Os valores do coeficiente de partição solo-solução de Freundilich ( $\left.\mathrm{K}_{\mathrm{F}}\right)$, o fator de linearidade (n), o coeficiente de partição solo-solução de Langmuir $\left(K_{L}\right)$ e capacidade máxima de sorção $\left(\mathrm{S}_{\mathrm{m}}\right)$, foram obtidos por regressão não-linear. Os resultados das regressões são apresentados na tabela (5). 
Tabela 5 - Parâmetros de sorção de Freündlich, Langmuir e Isoterma Linear

\begin{tabular}{|c|c|c|c|c|c|}
\hline Prof. (cm) & & $0-20$ & $20-40$ & $40-60$ & $60-80$ \\
\hline \multirow{2}{*}{ Linear } & $\mathrm{K}_{\mathrm{D}}\left(\mathrm{L} \cdot \mathrm{kg}^{-1}\right)$ & 1,94 & 1,32 & 1,57 & 1,41 \\
\hline & $\mathrm{R}^{2}$ & 0,97 & 0,97 & 0,95 & 0,99 \\
\hline \multirow{4}{*}{ Freundlich } & $\mathrm{K}_{F}\left(\mathrm{~kg}^{-1} \mathrm{mg}^{\left.1-1 / n \mathrm{~L}^{1 / n}\right)}\right.$ & 7,59 & 0,18 & 0,09 & 0,74 \\
\hline & $\mathrm{n}$ & 0,56 & 1,62 & 1,89 & 1,20 \\
\hline & $\mathrm{R}^{2}$ & 0,99 & 0,99 & 0,98 & 0,99 \\
\hline & $\mathrm{K}_{\mathrm{L}}$ & 0,046 & $-7,6 \mathrm{E}-05$ & $6,37 \mathrm{E}-05$ & $-1,4 \mathrm{E}-5$ \\
\hline \multirow[t]{2}{*}{ Langmuir } & $\mathrm{S}_{\mathrm{m}}\left(\mathrm{mg} \cdot \mathrm{L}^{-1}\right)$ & 86,98 & $1,7 \mathrm{E} 4$ & $2,4 \mathrm{E} 4$ & $1, \mathrm{OE}^{4}$ \\
\hline & $\mathrm{R}^{2}$ & 0,999 & 0,97 & 0,94 & 0,99 \\
\hline
\end{tabular}

Figura 4 - Isotermas de sorção da sulfadiazina para os solos das 4 profundidades de solo: a) 0 - $20 \mathrm{~cm}$; b) $20-40 \mathrm{~cm}$; c) $40-60 \mathrm{~cm}$; d) $60-80 \mathrm{~cm}$

a)

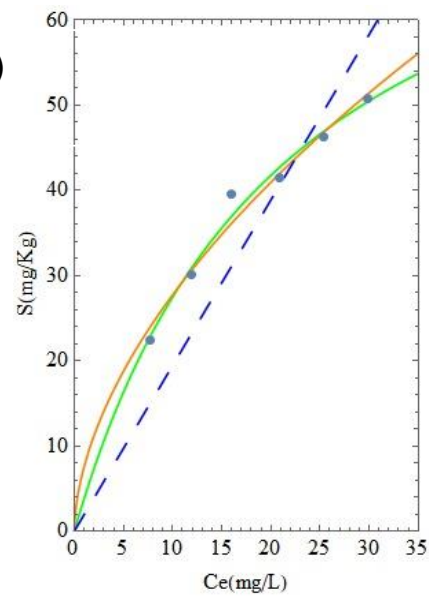

c)

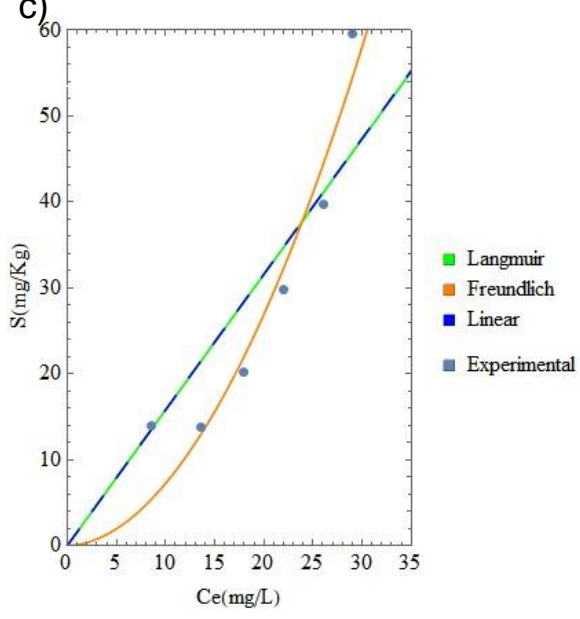

De acordo com as curvas da isotermas da Figura (4), notase que a sorção da sulfadiazina apresentou comportamentos distintos entre os solos estudados.

Entretanto os valores de $K_{D}$ obtidos do ajuste pelo modelo linear, pouco variaram entre os quatro tipos de solos $\left(\mathrm{K}_{\mathrm{D}}=\right.$

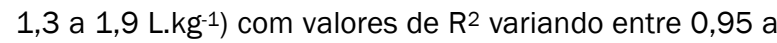
0.99. Esses valores estão condizentes com trabalhos anteriores que verificaram a adequação das isotermas da SDZ pelo modelo linear. Por exemplo, Conde-Cid et al. (2019a) obtiveram em um estudo de sorção da SDZ com b)
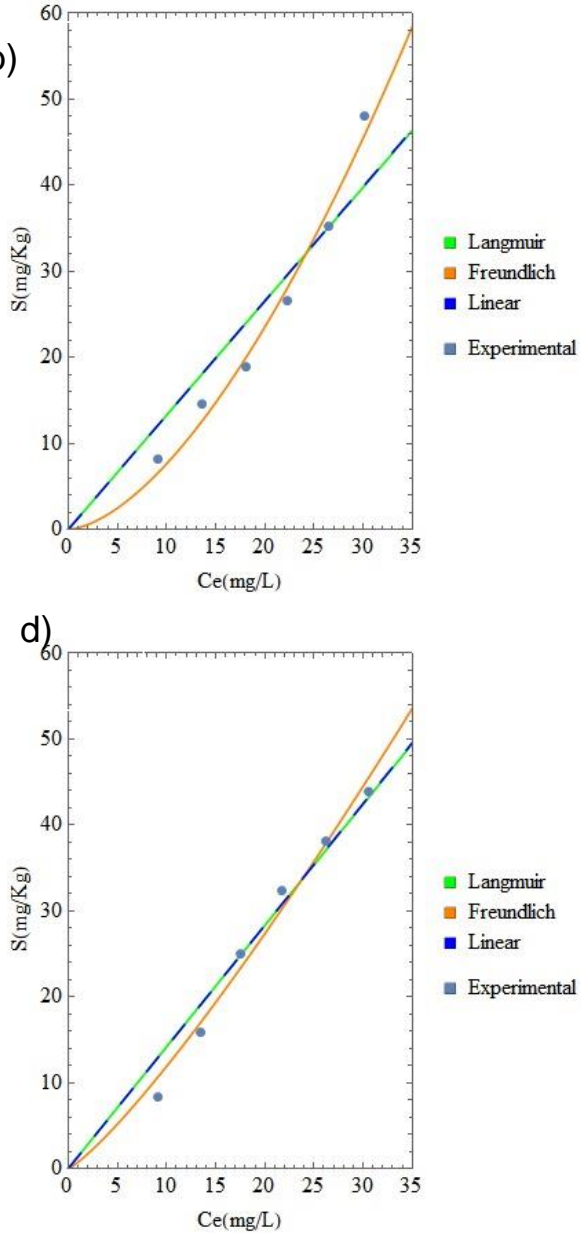

50 diferentes de solos, bom ajuste linear com valores de $\mathrm{R}^{2}$ entre 0,86 e 0,99 e valores médios de $\mathrm{K}_{\mathrm{D}}$ de 1,6 L. $\mathrm{kg}^{-1}$. Shen et al. (2018) obtiveram valores de $K_{D}$ de 1,22 L. kg-1 para sorção de SDZ e $K_{D}=1,23$ L.kg-1 $^{-1}$ do sulfametoxazol (SMX) para um solo com aproximadamente $16 \%$ de argila.

Na literatura são reportados vários trabalhos em que o modelo linear tem sido utilizado para descrever a sorção de várias sulfonamidas (DORETTO et al., 2014; PEREIRALEAL et al., 2013; SHEN et al., 2018; CONDE-CID et al., 2019a; CONDE-CID et al., 2019b). Nesses casos, os valo- 
res de $K_{D}$ são confiáveis para representar o potencial de sorção do contaminante.

Analisando o ajuste dos dados experimentais do modelo de Freundlich, observa-se comportamento bastante divergente para os solos das quatro profundidades. Esse modelo embora apresente valores de $\mathrm{R}^{2}$ próximo do ideal (01) todos os solos estudados, os valores de $\mathrm{K}_{\mathrm{F}}$ variaram consideravelmente. Por exemplo, o $\mathrm{K}_{\mathrm{F}}$ do solo de $0-20 \mathrm{~cm}$ foi muito superior ao demais $\left(7,59 \mathrm{~kg}^{-1} \mathrm{mg}^{1-1 / \mathrm{n}^{1 / n}}\right)$ e para o solo de $40-60 \mathrm{~cm}$, o valor foi bastante reduzido $(0,091 \mathrm{~kg}$ $\left.{ }^{1} \mathrm{mg}^{1-1 / n} \mathrm{~L}^{1 / \mathrm{n}}\right)$. Nota-se, também, que os valores do expoente de Freundilich ( $n$ ) se afastam bastante do valor de tendência linear para esses dois casos, para o solo 0$20 \mathrm{~cm}$ o $\mathrm{n}$ foi de 0,56 e para o solo $40-60 \mathrm{~cm}$ o $\mathrm{n}$ foi de 1,89 . Isso pode indicar que a isoterma da SDZ para esses solos é de tendência linear. 0 solo que apresentou $n$ mais próximo de 1, indicando uma sorção com tendências lineares, é o de $60-80 \mathrm{~cm}(n=1,2) \mathrm{com} \mathrm{K}_{F}$ igual a $0,74(\mathrm{~kg}$ $\left.{ }^{1} \mathrm{mg}^{1-1 / n} L^{1 / n}\right)$.

O modelo de Freundlich comumente é utilizado para estudos de isotermas de sulfonamidas. Por exemplo, Park e Huwe (2016) verificaram que as isotermas de Freundlich representaram adequadamente a sorção de três antibióticos do tipo sulfonamidas. Os estudos de sorção promovidos por Doretto e Rath (2013), realizados em quatro solos, mostraram que a sorção da sulfadiazina se ajustou ao modelo de Freundlich. No entanto, a sulfadiazina foi sorvida em quatro diferentes tipos de solos: N1 (arenoso), N2 (argiloso), S1 (argiloso) e S2 (argiloso).

Conde-Cid et al. (2019a) e Shen et al. (2018) utilizaram os modelo linear e de Freundlich para ajustar seus dados experimentais e obtiverem valores de $\mathrm{K}_{D}$ e $\mathrm{K}_{F}$ na mesma ordem de grandeza, sem grandes desvios. Isso pode indicar de maneira geral que o desempenho do modelo de Freundlich não foi adequado para as isotermas dos solos estudados. A variação nos valores de $\mathrm{K}_{\mathrm{F}}$ entre os solos das quatro profundidades naturalmente são atribuídas a diferentes propriedades físico-químicas dos solos, porém, de acordo com a Tabela (2), não houveram diferenças significativas entre os teores de argila, pH e matéria orgânica que justifique a diferença entre os valores de $\mathrm{K}_{\mathrm{F}}$ dos solos de 0-20 e 40-60 cm. Indicando que para os solos de todas as profundidades os valores de $\mathrm{K}_{\mathrm{D}}$ tendem a ser semeIhantes.

De maneira geral, a sorção da SDZ é diretamente influencianda pelas propriedades físico-químicas do solo. Por exemplo, Leal et al. (2013) verificaram que minerais de argila são um fator importante na influência da sorção das sulfonamidas e Park \& Huwe (2016), em sua análise do coeficiente de sorção $\left(K_{D}\right)$ para três antibióticos da família das sulfonamidas, a sorção ocorreu mais facilmente para o pH mais baixo. Vários experimentos sobre a sorção de antibióticos apontaram nos últimos anos uma relação direta entre a quantidade de $\mathrm{MO}$ e o grau de sorção dos antibióticos (YU, et al; 2015). Conforme Thiele-Bruhn (2003), quanto maior for o COT no solo, maior será a sorção das sulfonamidas. Doretto e Rath (2013) reiteram que solos muito argilosos adsorvem melhor a SDZ que solos arenosos.

Os valores da capacidade máxima de sorção $(\mathrm{Sm})$ dos ajustes pelo modelo de Langmuir indicam que para os solos das profundidades 20-40; 40-60; $60-80 \mathrm{~cm}$ esse modelo não apresentou um bom desempenho, apesar dos valore de $\mathrm{R}^{2}$ entre 0,94 a 0,99 esse modelo não tem sentido físico para as isotermas desses solos. Apenas para o solo de 0-20 cm o modelo de Langmuir apresenta um desempenho razoável com valores de $\mathrm{K}_{\mathrm{L}}=0,046 \mathrm{mg} \cdot \mathrm{L}^{-1}$ e $\mathrm{Sm}=$ 86,98 mg.kg-1.

A maioria dos estudos sobre as isotermas das sulfonamidas focam em sua adequação aos modelos lineares e de Freundlich. Contudo, Białk-Bielińska et al. (2012) observaram que as isotermas de Langmuir também se mostraram adequadas para descrever a sorção de duas sulfonamidas, a sulfaguanidina (SGD) e a sulfadimetoxina (SDM) nos três solos investigados. Para tal estudo, os três modelos (linear, de Freundlich e Langmuir) apresentaram coeficientes de regressão maiores que 0,92. Portanto, eles concluíram que a adequação das isotermas de Langmuir, para descrever o comportamento da sorção das sulfonamidas investigadas, pode ser explicada pela baixa concentração do contaminante usada nos experimentos ou provavelmente pela homogeneidade dos sítios de interações.

Nesse estudo o modelo linear é o que apresentou melhor desempenho e não houve diferença significativa entre os valores de $K_{D}$ refletindo a semelhança das propriedades físico-químicas dos solos, que pouco variaram. De maneira geral os baixos valores dos coeficientes de partição solosolução dos ajustes aos modelos de isotermas indicam uma fraca sorção entre a moléculas do SDZ e a matriz sólida dos solos, acarretando em alta mobilidade desses compostos e elevando o risco de contaminação das águas subterrâneas. Baixos valores do coeficiente de partição solo-solução são relacionados com alta mobilidade do poluente da SDZ em vários trabalhos (SUKUL et al., 2008; Doreto e Rath, 2013; SRINIVASAN, SARMAH, 2014; SHEN et al. 2018; CONDE-SID et al., 2019a)

\section{CONCLUSÃO}

A sorção da sulfadiazina no solo é uma importante informação para a determinação do potencial de mobilidade e retenção desse poluente emergente no solo e para a estimativa do risco de contaminação das águas subterrâneas. 
Esse estudo ocorreu de um perfil de solo urbano da cidade do recife.

De acordo com a cinética de sorção o equilíbrio foi alcançado com 24 horas de interação e o modelo cinético de segunda ordem foi o mais adequado para representar a sorção da SDZ em todos os solos do perfil.

As isotermas de sorção foram melhor representadas pelo modelo linear, com valores de $\mathrm{R}^{2}$ satisfatórios e $\mathrm{K}_{\mathrm{D}}=1,3$ a 1,9 L.kg-1. Os modelos não lineares não apresentaram desempenho adequado. Os coeficientes de sorção relativamente baixos para SDZ encontrados neste estudo indicam sua fraca interação com os sítios de ligação nos solos. Isto sugere que este antimicrobiano tem potencial para atingir águas superficiais e subterrâneas.

\section{AGRADECIMENTOS}

Este trabalho contou como apoio do projeto intitulado Transferência de Água e de Misturas de Poluentes Reativos em Solos Antropizados (APQ - 436875/2018-7) apoiado pelo Conselho Nacional de Desenvolvimento Científico e Tecnológico (CNPq) - Brasil.

\section{REFERÊNCIAS}

BU, Q., WANG, B., HUANG, J., DENG, S., \& YU, G. Pharmaceuticals and personal care products in the aquatic environment in China: a review. Journal of hazardous materials, 2013, v.262, p.189-211.

BIAŁK-BIELIŃSKA, A., MASZKOWSKA, J., MROZIK, W., BIELAWSKA, A., KOŁODZIEJSKA, M., PALAVINSKAS, R., STEPNOWSKI, P., KUMIRSKA, J. Sulfadimethoxine and sulfaguanidine: their sorption potential on natural soils. Chemosphere, v. 86, n. 10, p.1059-1065, 2012.

CARVALHO, I.T., SANTOS, L. Antibiotics in the aquatic environments: a review of the European scenario. Environment International, v.94, p.736-757, 2016.

CONDE-CID, M., NÓVOA-MUÑOZ, J. C., FERNÁNDEZ-SANJURJO, M. J., NÚÑEZ-DELGADO, A., ÁLVAREZ-RODRÍGUEZ, E., \& ARIAS-ESTÉVEZ, M.. Pedotransfer functions to estimate the adsorption and desorption of sulfadiazine in ag ricultural soils. Science of The Total Environment, 2019a.

CONDE-CID, M., FERNÁNDEZ-CALVIÑO, D., FERNÁNDEZSANJURJO, M. J., NÚÑEZ-DELGADO, A., ÁLVAREZRODRÍGUEZ, E., \& ARIAS-ESTÉVEZ, M. Adsorption/desorption and transport of sulfadiazine, sulfachloropyridazine, and sulfamethazine, in acid agricultural soils. Chemosphere, 2019b.

DAN, A., YANG, Y., DAI, Y. N., CHEN, C. X., WANG, S. Y., \& TAO, R. Removal and factors influencing removal of sulfonamides and trimethoprim from domestic sewage in constructed wetlands. Bioresource technology, v.146, p.363370,2013
DORETTO, K. M., RATH, S. Sorption of sulafadizine on Brazilian soils, Chemosphere, 2013. v. 90, n. 6, p.2027-2034.

EMBRAPA - Empresa Brasileira de Pesquisa Agropecuária. Manual de métodos de análises de solo. [S.I]. 1997.

GOBEL, A., MCARDELL, C. S., JOSS, A., SIEGRIST, H., GIGER, W. Fate of sulfonamides, macrolides, and trimethoprim in different wastewater treatment technologies. Science of the Total Environment, v.372, p. 361-388, 2007.

HE, K., SOARES, A.D., ADEJUMO, H. L. Detection of a wide variety of human and veterinary fluoroquinolone antibiotics in municipal wastewater and wastewater-impacted surface water. Journal Pharmaceutical and Biomedical Analysis, 2015. v.106, p.136-143.

HO, Y. S., \& MCKAY, G. Pseudo-second order model for sorption processes. Process Biochemistry, v.34. p.451465, 1999.

HU, L. MARTIN, H.M. STRATHMANN T.J. Oxidation kinetics of antibiotics during water treatment with potassium permanganate. Environental Science Technology, v.44, p.6416-6422, 2010.

IBGE - Instituto Brasileiro de Geografia e Estatística. Atlas de saneamento Rio de Janeiro: IBGE, 2011. 268p, 2011.

IBGE - Instituto Brasileiro de Geografia e Estatística. Sidra (a). Banco de dados agregados. População censo 2010. Tabela 3107. Disponível em: http://www.sidra.ibge.gov.br/bda/tabela/lstabl.asp?z=t\&c=1378. Acesso em: Set.2013.

JOHNSON, A.C., KELLER, V., DUMONT, E., SUMPTER, J.P. Assessing the concentrations and risks of toxicity from the antibiotics ciprofloxacin, sulfamethoxazole, trimethoprim and erythromycin in European rivers. Science of the Total Environment, v.51, p.747, 2015.

KÜMMERER, K. Antibiotics in the aquatic environment-a review-part I. Chemosphere, v.75, n. 4, p.417-434, 2009.

LEAL, R. M. P., ALLEONI, L. R. F., TOMISIELO, V., L. REGITANO, J. B. Sorption of fluoroquinolones and sulfonamides in 13 Brazilian soils. Chemosphere, v.92, p.979985, 2013.

LIU, P., WANG, Q., ZHENG, C., HE, C. Sorption of Sulfadiazine, Norfloxacin, Metronidazole and Tetracycline by granular actived carbom: Kinetics, Mechanisms and Isotherms. Water Air Soil Pollut, v. 228, n. 4, p.129, 2017.

MILFONT, M. L. B.; ANTONINO, A. C. D.; MARTINS, J. M. F.; NETTO, A. M., CORREA, M. M. Caracterização hidrodispersiva de dois solos do vale do rio São Francisco. Revista brasileira de ciências agrárias, v. 1, p.81-87, 2006.

PARK, J. Y.; HUWE, B. Effect of $\mathrm{pH}$ and soil structure on transport of sulfonamide antibiotics in agricultural soils. Environmental Pollution, v.213, p.561-570, 2016. 
PAVLOVIC, D. M., GLAVAC, A., GLUHAK, M., RUNJE, M. Sorption of albendazole in sediments and soils: Isotherms and Kinetics. Chemosphere, v.193, p.635-644, 2018.

PERISA, M., BABIC, S., SKORTIC, I., FROMEL, T., KNEPPER, T. P. Photodegradation of sulfonamides and their $\mathrm{N}^{4}$-acetylatd metabolites in water by simulated sunlight irradiation: kinetics and identification of photoproducts. Environmental Science and Pollution Research, v.20, p.89348946, 2013

PERUCHI, L. M., FOSTIER, A. H., RATH, S. Sorption of norfloxacin in soils: Analytical method, kinetics and Freundlich isotherms. Chemosphere, 2015. v.119, p.310-317.

RODRIGUEZ-MOZAZ, S., CHAMORRO, S., MARTI, E., HUERTA, B., GROS, M., SÀNCHEZ-MELSIÓ, A., BORREGO, C.M., BARCELÓ, D., BALCÁZAR, J.L. Occurrence of antibiotics and antibiotic resistance genes in hospital and urban wastewaters and their impact on the receiving river. Wate Ressources, v.69, p.234242, 2015.

SRINIVASAN, P., SARMAH, A. K. Dissipation of sulfamethoxazole in pasture soils as affected by soil and environmental factors. Science of the Total Environment, v.479480, p.284-291, 2014.

SHEN, G., ZHANG, Y., HU, S., ZHANG, H., YUAN, Z., ZHANG, $W$. Adsorption and degradation of sulfadiazine and sulfamethoxazole in an agricultural soil system under an anaerobic condition: Kinects and environmental risks. Chemosphere, v.194, p.266-274, 2018.

SUKUL, P., SPITELLER, M. Sulfonamides in the environment as veterinary drugs. Reviews of Environmental Contamination Toxicology, v.187, p.67-101, 2006.

SUKUL, P.; LAMSHÖFT, M.; ZÜHLKE, S.; SPITELLER, M. Sorption and desorption of sulfadiazine in soil and soil-manure systems. Chemosphere, v. 73, n. 8, p. 1344-1350, 2008.

TERNES, T., JOSS, A., OEHLMANN, J. Occurrence, fate, removal and assessment of emerging contaminants in water in the water cycle (from wastewater to drinking water). Water Resssources, v.72, p.1-2, 2015.
THIELE-BRUHN, S.J. Pharmaceutical antibiotics compounds in soil - a review. Journal of Plant Nutrition and Soil Science, v.166, p.145-167, 2003.

THIELE-BRUHN, S., SEIBICKE, T., SCHULTEN, H. R., \& LEINWEBER, P. Sorption of sulfonamide pharmaceutical antibiotics on whole soils and particle-size fractions. Journal of Environmental Quality, v.33, 4, p.1331-1342, 2004.

$\mathrm{Xu}, \mathrm{J} ., \mathrm{Xu}, \mathrm{Y}$., Wang, H. Occurrence of antibiotics and antibiotic resistance genes in a sewage treatment plant and its effluent-receiving river. Chemosphere, v.119, p.13791385, 2015.

ZANG, Y., HU, S., SHEN, G., YUAN, Z., ZHANG, W. Degradation kinetics and mechanism of sulfadiazine and sulfamethoxazole in an agricultural soil system with manure application. Science of the Total Environment, v. 607-608, p.1348-1356, 2017.

YANG, J., HE, M., WU, T., HAO, A., ZHANG, S., CHEN, Y., ZHOU, S., ZHEN, L., WANG, R., YUAN, Z., DENG, L. Sulfadiazine oxidation by permanganate: Kinetics, mechanistic, investigation and toxicity evaluation. Chemical Engineering Journal, v.349, p.56-65, 2018.

YAN, C., YANG, Y., ZHOU, J., LIU, M., NIE, M., SHI, H. Antibiotics in the surface water of the Yangtze Estuary: occurrence, distribution and risk assessment. Environmental Pollution, v.175, p.22, 2013.

YANEVA, Z., KOUMANOVA, B. Comparative modelling of mono- and dinitrophenols sorption on yellow bentonite from aqueous solutions. Journal Colloid Interf. Sci. v. 293, p.303-311, 2006.

YU, X.; ZHANG, L.; LIANG, M.; SUN, W. pH-dependent sulfonamides adsorption by carbon nanotubes with different surface oxygen contents. Chemical Engineering Journal, 279, p. 363-371, 2015. 